\title{
Predictors of Perioperative Vasoactive Drug Requirement During Retroperitoneal Adrenalectomy for Pheochromocytoma: A Retrospective Exploratory Study
}

Authors

Mikhail Alexeev $^{1}{ }^{\mathbb{D}}$, Oleg Kuleshov ${ }^{1}$, Elisei Fedorov², Kirill Gorokhov¹, Vladimir Rusakov², Tatiana Ionova ${ }^{3}$, Tatiana Nikitina ${ }^{3}$, Stefan Bornstein ${ }^{4}$, Sergey Efremov ${ }^{1}$ (D)

Affiliations

1 Department of Anesthesiology and Intensive Care, Saint-Petersburg State University Hospital, St. Petersburg, Russian Federation

2 Department of Endocrine Surgery, Saint-Petersburg State University Hospital, St. Petersburg, Russian Federation

3 Quality of Life and Monitoring Unit, Saint-Petersburg State University Hospital, St. Petersburg, Russian Federation

4 Department of Medicine III, University Hospital Carl Gustav Carus, Technische Universität Dresden, Dresden, Germany

Key words pheochromocytoma, transpulmonal thermodilution, vasoactive inotropic score, predictors vasopressor use

received 05.05 .2021

accepted after revision $\quad$ 15.09.2021

Bibliography

Horm Metab Res 2021; 53: 717-722

DOI 10.1055/a-1651-5975

ISSN 0018-5043

(c) 2021. Thieme. All rights reserved.

Georg Thieme Verlag KG, Rüdigerstraße 14,

70469 Stuttgart, Germany

Correspondence

Mikhail Alexeev

Department of Anesthesiology and Intensive Care

Saint-Petersburg State University Hospital

St. Petersburg

Russian Federation

Tel.: + 79218987926

Miha_Decor@mail.ru
Supplementary material is available under https:// doi.org/10.1055/a-1651-5975.

\section{ABSTRACT}

The aim of the present study was to test a hypothesis that baseline systemic vascular resistance index (SVRI) assessed by method of transpulmonary thermodilution predicts perioperative requirement for vasoactive drugs. The primary outcomes were: (1) peak vasoactive-inotropic score (VIS) and (2) peak dose of hypotensive drugs at any stage of surgery. The main exposure variable was baseline SVRI. Hemodynamics were retrospectively assessed by transpulmonary thermodilution in 50 adults who had undergone posterior retroperitoneal surgery for pheochromocytoma. Univariate linear regression analysis showed predictive value of SVRI on VIS [regression coefficient, $95 \% \mathrm{Cl} ; 0.024$ $(0.005,0.4), p=0.015]$. Other significant factors were the history of peak diastolic pressure, baseline MAP, baseline betablocker therapy, and history of coronary artery disease (CAD). After adjustment of SVRI for the history of CAD, its prognostic value became non-significant $[0.018(0.008,0.03), p=0.063$ and 29.6 $(19,40.2), p=0.007$ for SVRI and history of CAD, respectively]. Requirements of vasodilators were predicted by baseline adrenergic activity $[0.37(0.005,0.74), p=0.047]$. In conclusion, baseline SVRI is associated with perioperative requirement of vasopressor drugs, but history of CAD is a stronger prognostic factor for vasopressor support. Perioperative requirement in vasodilators is associated with baseline adrenergic activity.

\section{Introduction}

Anesthetic management for pheochromocytoma resection is a challenge for anesthesiologists [1]. The course of anesthesia is as- sociated with risks of both intraoperative hypertension during adrenal mobilization and severe hypotension in the post-resection period [2,3], which might be further related to an adverse clinical 
course, bleeding, and/or major vascular complications [4]. To prevent such serious adverse events, preoperative patient evaluation traditionally includes the prescription of alpha adrenoblockers $[2,3]$. However, the use of this approach varies among centers $[5,6]$. As no minor complication or critical incident (such as severe hypertension, hypotension, or need for vasoactive drugs) has been universally accepted [7-9], several attempts have been made to determine the predictors of unstable hemodynamics for this patient cohort [7, 8]. Such predictors include use of alphablockers [5], tumor size, preoperative maximum blood pressure, and level of preoperative metanephrines [7,8]. Additionally, there are currently no data on the central hemodynamics of the surgery and its possible predictive and clinical impact. Hence, this retrospective study aimed to analyze the perioperative hemodynamics of patients who had undergone posterior retroperitoneoscopic adrenalectomy and to determine the predictors of intraoperative hemodynamic instability.

\section{Patients and Methods}

\section{Ethics Approval}

This retrospective observational study was approved by local ethics committee of Saint-Petersburg State University Hospital (protocol number 03/21), with requirement for informed consent waived due to the retrospective design of the study. The aim of present study was to test a hypothesis that baseline systemic vascular resistance index (SVRI) predicts perioperative requirement for vasoactive drugs.

\section{Study population}

Inclusion criteria: patients who (1) were aged 18 years and older and (2) had undergone posterior retroperitoneoscopic adrenalectomy for pheochromocytoma between October 2015 and February 2020. Exclusion criteria: patients with (1) bilateral tumor localization, (2) unavailable or insufficient retrospective central hemodynamic data, and (3) need for conversion to open surgery. Data were extracted from electronic medical records.

\section{Outcomes}

The primary outcomes were: (1) peak vasoactive-inotropic score (VIS) and (2) peak dose of hypotensive drugs, expressed as multiplicity of exceeding the upper limit of the recommended starting doses.

The VIS was determined using the following formula: VIS = dopamine dose $(\mu \mathrm{g} / \mathrm{kg} / \mathrm{min})+$ dobutamine dose $(\mu \mathrm{g} / \mathrm{kg} / \mathrm{min})+100 \times$ epinephrine dose $(\mu \mathrm{g} / \mathrm{kg} / \mathrm{min})+10 \times$ milrinone dose $(\mu \mathrm{g} / \mathrm{kg} / \mathrm{min})+10$ $000 \times$ vasopressin dose $(\mathrm{U} / \mathrm{kg} / \mathrm{min})+100 \times$ norepinephrine dose $(\mu \mathrm{g} /$ $\mathrm{kg} / \min )[10,11]$.

The upper limits of the recommended starting doses were $0.25 \mu \mathrm{g} / \mathrm{kg} / \mathrm{min}$ for nitroglycerine and $50 \mu \mathrm{g} / \mathrm{kg} / \mathrm{min}$ for esmolol.

\section{Exposure variables}

The main exposure variable was baseline SVRI measured by transpulmonary thermodilution. The secondary exposure variables were baseline MAP, GEDI, duration of administration and daily dose of alpha-blockers, chronic administration of beta-blockers, histo- ry of coronary artery disease, adrenergic activity, peak prehospital systolic and diastolic pressures, tumor size and signs of tumor decay on tomography.

\section{Clinical and hemodynamic evaluation}

Patients were hospitalized at least one day before the surgery. Pheochromocytoma was diagnosed in the prehospital period based on clinical signs, contrast-enhanced computed tomography, and laboratory evaluation of adrenergic activity (catecholamine metabolites). Due to the variability in the methods of baseline catecholamine metabolite assessment (metanephrine and/or normetanephrine levels in blood and/or urine), the levels were expressed in multiplicity of exceeding the upper limit of normal values. All patients continued their regular medications till the day of surgery.

Patients were operated under general anesthesia. Anesthesia was induced with $2 \mu \mathrm{g} / \mathrm{kg}$ fentanyl, $2 \mathrm{mg} / \mathrm{kg}$ propofol, and $0.6 \mathrm{mg} /$ $\mathrm{kg}$ rocuronium and maintained with desflurane in 1-2 $1 / \mathrm{min}$ of fresh gas with $50 \%$ oxygen fraction. Intraoperative monitoring included electrocardiography, body temperature, end-tidal $\mathrm{CO}_{2}$, arterial blood gas levels, bispectral index, pulse oximetry, and continuous monitoring of extended hemodynamics including pulse contour cardiac output through transpulmonary thermodilution (PiCCOplus system, Pulsion Medical Systems, Munich, Germany). The following hemodynamic parameters were evaluated: systolic arterial pressure, diastolic arterial pressure, mean arterial pressure (MAP), systemic vascular resistance index (SVRI), cardiac index $(\mathrm{CI})$, and global end diastolic volume index at the following stages: 1 ) before anesthesia induction, 2) after anesthesia induction, 3) prone positioning, 4) during surgical mobilization of the adrenal gland, 5) just after ligation of the central gland vein, 6) end of surgery, 7) supine positioning, and 8) end of anesthesia.

All surgeries were performed by a single surgeon using the posterior retroperitoneoscopic approach described by Walz et al. [12].

Perioperative hypotension was treated using intravenous fluids and/or infusion of catecholamines (norepinephrine, epinephrine, dopamine, or phenylephrine). Hypertension was treated using esmolol and/or nitroglycerine. The drugs of choice were at the discretion of the anesthesiologist.

All patients were transmitted to the intensive care unit after the surgery. The criteria for extubation were stable hemodynamics without the infusion or with the progressive lowering dose of vasoactive drugs, normal lactate levels, recovery of spontaneous breathing, and conscious and adequate muscle tone.

\section{Statistics}

Since this was an observational audit study, no formal power analysis was performed. Categorical data were presented as numbers and percentages. Continuous variables were presented as medians and 25th and 75th percentiles. Friedman's test was used for comparing the hemodynamic variables at all stages. Normality was tested using Shapiro-Wilk normality test. If the results of the Friedman's test were significant, appropriate post hoc multiple comparisons were performed. Linear regression analysis was performed to assess the relationship between VIS or dose of hypotensive drugs and exposure variables. To test for multicollinearity, the correlations between independent variables were calculated using the pairwise Pearson's correlation coefficient. Results were expressed 
as regression coefficients with $95 \%$ confidence intervals and coefficients of determination $R^{2}$. $p$-Values of less than 0.05 were used as a cut-off point for statistical significance and all statistical tests were two-sided. The statistical analyses were performed using MedCalc Statistical Software version 19.0.5 (MedCalc Software bvba, Ostend, Belgium).

- Table 1 Demographic data.

\begin{tabular}{|c|c|}
\hline Variable & Value \\
\hline Number of patients & 50 \\
\hline Female gender & $40(80 \%)$ \\
\hline Age, years & $42(34 ; 56)$ \\
\hline Body mass index, $\mathrm{kg} / \mathrm{m}^{2}$ & $23.8(21.01 ; 25.98)$ \\
\hline Alpha-blockers & $38(76 \%)$ \\
\hline Beta-blockers & $22(44 \%)$ \\
\hline Other antihypertensives & $14(28 \%)$ \\
\hline Coronary artery disease & $11(22 \%)$ \\
\hline Tumor size, $\mathrm{cm}^{3}$ & $69.19(53.92 ; 110.95)$ \\
\hline Adrenergic activity $^{*}$ & $4.86(1.96 ; 7.7)$ \\
\hline ASA 2 & $5(10 \%)$ \\
\hline ASA 3 & $45(90 \%)$ \\
\hline Duration of anesthesia, min & $140(125 ; 183.75)$ \\
\hline Duration of surgery, min & $90(70 ; 115)$ \\
\hline ICU ventilation time, min & $\begin{array}{l}5(10 \%) \\
100(76.25 ; 120)\end{array}$ \\
\hline Need inotropic support after anesthesia & $21(42 \%)$ \\
\hline Duration time, hours & $5(3 ; 18.25)$ \\
\hline Norepinephrine use & $34(68 \%)$ \\
\hline Epinephrine & $26(52 \%)$ \\
\hline Dopamine & $21(42 \%)$ \\
\hline Phenilephrine & $1(2 \%)$ \\
\hline VIS * & $29.5(11 ; 50)$ \\
\hline Nitroglycerine use & $42(84 \%)$ \\
\hline Esmolol use & $34(68 \%)$ \\
\hline Hypotensive drugs use ${ }^{* * *}$ & $22(10.9 ; 46.7)$ \\
\hline Intraoperative infusion, ml & $2150(1700 ; 2500)$ \\
\hline Urine output, ml & $600(300 ; 800)$ \\
\hline ICU stay, nights & $1(1 ; 1)$ \\
\hline Hospital stay, nights & $3(3 ; 4)$ \\
\hline Signs of tumor decay ${ }^{* * * *}$ & $13(26 \%)$ \\
\hline \multicolumn{2}{|c|}{$\begin{array}{l}{ }^{*} \text { Multiplicity of exceeding the upper limit of normal values of } \\
\text { catecholamines as they are or their metabolites in blood or urine } \\
\text { depending on the method of measuring. }{ }^{* *} \text { Vasoactive Inotropic } \\
\text { Score }(\mathrm{VIS}) \text { is equal to dopamine dose }(\mu \mathrm{g} / \mathrm{kg} / \mathrm{min})+\text { dobutamine } \\
\text { dose }(\mu \mathrm{g} / \mathrm{kg} / \mathrm{min})+100 \times \text { epinephrine dose }(\mu \mathrm{g} / \mathrm{kg} / \mathrm{min})+10 \times \text { milri- } \\
\text { none } \mathrm{dose}(\mu \mathrm{g} / \mathrm{kg} / \mathrm{min})+10000 \times \text { vasopressin dose }(\mathrm{U} / \mathrm{kg} / \\
\text { min) }+100 \times \text { norepinephrine dose }(\mu \mathrm{g} / \mathrm{kg} / \mathrm{min}) .{ }^{* *} \text { Multiplicity of } \\
\text { exceeding the upper limit of recommended start doses (peak used } \\
\text { dose of nitroglycerine } / 0.25 \mu \mathrm{g} / \mathrm{kg} / \mathrm{min}+\text { peak used dose of } \\
\text { esmolol } / 50 \mu \mathrm{g} / \mathrm{kg} / \mathrm{min}) .{ }^{* *} \text { Signs of partial tumor necrosis by } \\
\text { computed tomography. }\end{array}$} \\
\hline
\end{tabular}
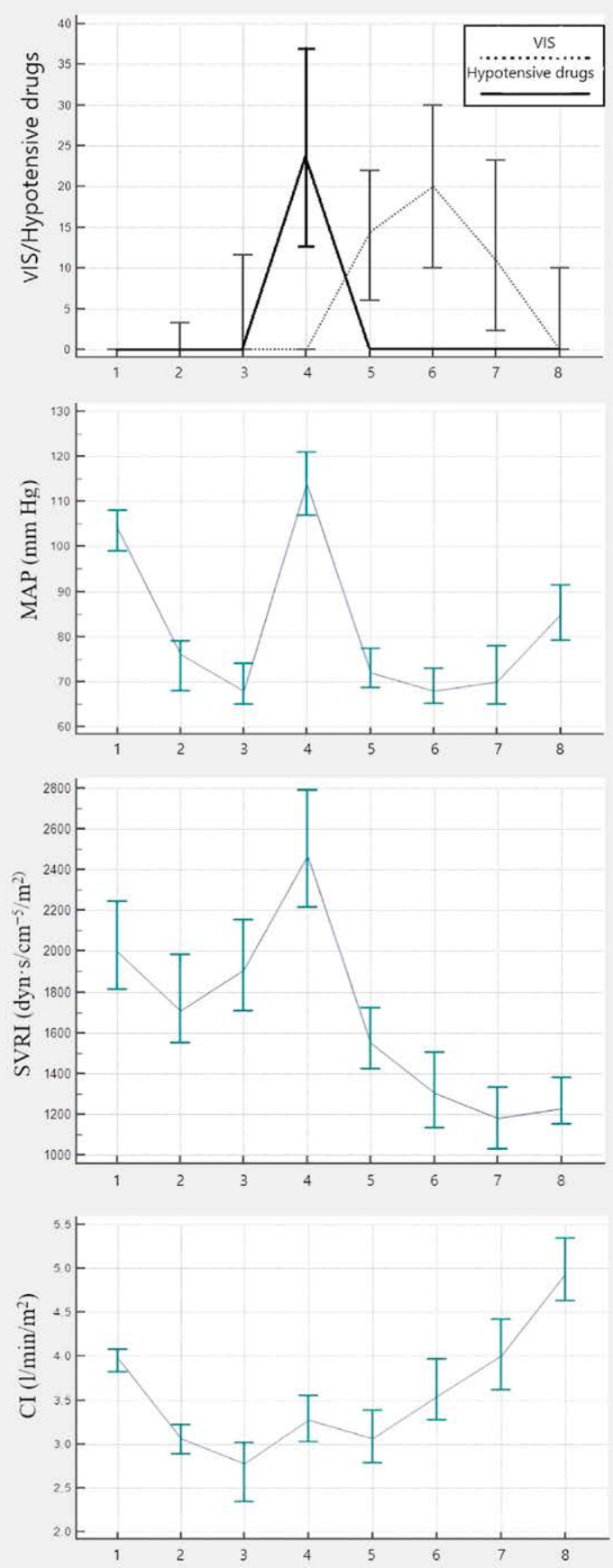

- Fig. 1 The relationship between key parameters of central hemodynamics and requirement of vasoactive drugs during the following stages of surgery: (1) before induction of general anesthesia; (2) after induction of general anesthesia; (3) prone position; (4) mobilization of adrenal gland; (5) ligation of central vein of adrenal gland; (6) end of surgery; (7) supine position; and (8) end of anesthesia. Vasoactive Inotropic Score (VIS) is equal to dopamine dose $(\mu \mathrm{g} / \mathrm{kg} /$ $\min )+$ dobutamine dose $(\mu \mathrm{g} / \mathrm{kg} / \mathrm{min})+100 \times$ epinephrine dose $(\mu \mathrm{g} /$ $\mathrm{kg} / \mathrm{min})+10 \times$ milrinone dose $(\mu \mathrm{g} / \mathrm{kg} / \mathrm{min})+10000 \times$ vasopressin dose $(\mathrm{U} / \mathrm{kg} / \mathrm{min})+100 \times$ norepinephrine dose $(\mu \mathrm{g} / \mathrm{kg} / \mathrm{min})$. Hypotensive drugs are expressed as a multiplicity of exceeding the upper limit of recommended start doses (peak used dose of nitroglycerine $/ 0.25 \mathrm{mcg} / \mathrm{kg} / \mathrm{min}$ + peak used dose of esmolol $/ 50 \mu \mathrm{g} / \mathrm{kg} / \mathrm{min}$ ). MAP: Mean arterial pressure, $\mathrm{mmHg}$; $\mathrm{Cl}$ : Cardiac index, I/min/m²; SVRI: System vascular resistance index, dyn $\cdot \mathrm{s} / \mathrm{cm}^{-5} / \mathrm{m}^{2}$. 


\section{Results}

Overall, 196 patients ( $80 \%$ women, average age 42 ) with pheochromocytoma who had undergone retroperitoneoscopic adrenalectomy between October 2015 and February 2020 were assessed for eligibility. Six patients were excluded due to bilateral tumor localization, six due to need for conversion to laparotomy, and data on transpulmonary thermodilution were not available in 134 cases. Finally, 50 patients were included in the study. Baseline and perioperative clinical data are presented in $>$ Table 1.

\section{Analysis of central hemodynamics}

A detailed description of the central hemodynamic parameters is presented in Table S1. Trajectories of intraoperative changes in VIS, hypotensive drugs, MAP, SVRI, and $\mathrm{Cl}$ are presented in — Fig. 1.

\section{Regression analysis}

The correlation chart exploring the multicollinearity of the variables was outlined before the linear regression analysis (Table S2). In accordance with the univariate regression analysis, VIS was significantly associated with baseline SVRI $[0.024(0.005,0.04)$, $p=0.015]$. Other significant factors included history of peak diastolic pressure, baseline MAP, baseline betablocker therapy and history of coronary artery disease. However, after adjustment for the history of coronary artery disease, prognostic value of SVRI lost sig- nificance ( $\triangleright$ Table 2 ). The only significant factor to predict hypotensive drug requirement according to univariate regression analysis was adrenergic activity $[0.37(0.0047,0.74), p=0.047]$. There were no fatalities or cases of myocardial infarction or stroke. In-hospital outcomes are presented in Table S3.

\section{Discussion}

In accordance with the results of this retrospective study, baseline SVRI predicts perioperative peak VIS. Furthermore, this retrospective study indicates the differential predictive value of preoperative factors on the risk of intraoperative requirement in hypotensive drugs and vasopressors.

Anesthetic management for posterior retroperitoneoscopic pheochromocytoma resection is associated with multidirectional but anticipated hemodynamic changes according to the stage of surgery and anesthesia. Previously published studies primarily focused on incidence of hemodynamic changes during the mobilization and resection of the adrenal gland [6,7] and requirement of sympathomimetic support after surgery $[13,14]$. In our opinion, isolated arterial pressure (AP) values do not reflect the actual hemodynamic condition during anesthesia and hence, should be assessed in conjunction with concomitantly used vasoactive agents. Thus, we decided to analyze indexed parameters as markers of perioperative hemodynamic stability. Vasopressor support, evaluat-

- Table 2 Univariate and multivariate regression analysis for perioperative VIS predictors.

\begin{tabular}{|c|c|c|c|c|}
\hline \multirow[t]{2}{*}{ Variable } & \multicolumn{2}{|c|}{ Univariate analysis } & \multicolumn{2}{|c|}{ Multivariate analysis * } \\
\hline & B $(95 \% \mathrm{CI})$ & $\mathbf{p}$ & B (95\% Cl) & $\mathbf{p}$ \\
\hline Baseline SVRI. dyn $\cdot s / \mathrm{cm}^{-5} / \mathrm{m}^{2}$ & $0.02424(0.004981 ; 0.04349)$ & 0.015 & $0.0176(0.0083 ; 0.0269)$ & 0.0630 \\
\hline Alphablockers dose, mg & $1.7354(-0.4158 ; 3.8867)$ & 0.111 & - & - \\
\hline Duration of admission of alphablockers, weeks & $-0.04697(-0.3219 ; 0.2279)$ & 0.733 & - & - \\
\hline Betablockers & $20.8766(2.1660 ; 39.5873)$ & 0.03 & - & - \\
\hline Baseline MAP, mm Hg & $0.7939(0.1926 ; 1.3951)$ & 0.011 & - & - \\
\hline CAD & 35.0932 (13.8406; 56.3459) & 0.002 & 29. 5854 (18.99; 40.18) & 0.0071 \\
\hline Adrenergic activity & $-0.1827(-0.7322 ; 0.3669)$ & 0.507 & - & - \\
\hline GEDI, $\mathrm{ml} / \mathrm{m}^{2}$ & $0.02282(-0.04400 ; 0.08963)$ & 0.496 & - & - \\
\hline Peak SAD (prehospital stage), mm Hg & $0.2110(-0.02569 ; 0.4477)$ & 0.079 & - & - \\
\hline Peak DAD (prehospital stage), mm Hg & 0.6095 (0.04462; 1.1743$)$ & 0.035 & - & - \\
\hline Tumor size, $\mathrm{cm}^{3}$ & $\begin{array}{l}0.00001552(-0.00004054 \\
0.00007159)\end{array}$ & 0.58 & - & - \\
\hline Signs of tumor decay & $2.5534(-17.2126 ; 22.3193)$ & 0.796 & - & - \\
\hline Volume expand before operation, ml & $-0.003418(-0.01733 ; 0.01050)$ & 0.624 & - & - \\
\hline \multicolumn{5}{|c|}{ 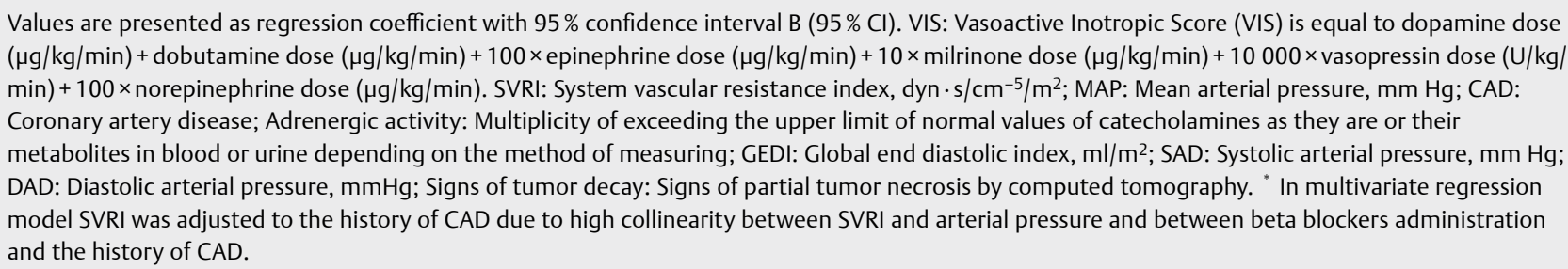 } \\
\hline
\end{tabular}


ed using the VIS score, has been associated with adverse long-term outcomes $[10,11]$. Unfortunately, we were unable to find a similar index for hypotensive drugs, and hence, we consider to express their doses as multiplicity of exceeding the upper limit of the recommended starting doses. We believe that absolute values of arterial pressure without evaluating the contribution of vasoactive drugs and extended hemodynamics, during the resection of pheochromocytoma, do not reflect the actual hemodynamic profile. Thus, despite a nonsignificant difference between MAP at baseline and after the mobilization of the adrenal gland, it was maintained by the infusion of hypotensive drugs in high doses. Accordingly, we decided to neglect intraoperative AP as a measure of perioperative hemodynamics, focusing on combination of extended hemodynamic data with the need for vasoactive drugs during anesthesia. Despite that hemodynamic assessment with transpulmonary thermodilution is not routine practice for the anesthetic management of pheochromocytoma, it has been well validated in various clinical situations and in contrast to AP may be more valuable for the assessment of hemodynamics and blood supply of the vital organs $[15,16]$. However, data of using this method for the adrenal gland surgery are lacking. A distinctive analysis of SVRI and CI showed that the stabilization of MAP after vein ligation was accompanied by decreasing afterload and a compensatory increase in heart work with significant vasoactive support. which may increase myocardial oxygen consumption. In some cases of compromised heart function, the result can include myocardial ischemia and cardiac complications $[15,16]$.

Other studies $[2,4,5]$ have traditionally evaluated MAP and/or duration of sympathomimetic support after the surgery [6, 7]. Existing data show that the dose of alphablockers does not predict perioperative AP $[4,5,17]$, but affects the need for vasoactive agents after the surgery [17]. The role of preoperative alphablockade has been discussed $[2,4,17,19]$. Our findings are partly concordant with these data; neither the baseline administration of alphablockers nor the time of their consumption has a significant effect on the VIS or need for hypotensive drugs during surgery. However, our data do not support the association between preoperative alphablockers and the postoperative need of vasoactive drugs. Other previously recognized predictors of perioperative adverse hemodynamic incidents are adrenergic activity and tumor size $[7,8]$. However, we did not find any effect of these parameters on VIS; although, adrenergic activity predicted the requirement of hypotensive drugs, which is consistent with the findings of previous studies $[7,8]$.

In terms of the clinical relevance of the presented data, it can be supposed that transpulmonary thermodilution should not be used routinely in any patient with pheochromocytoma, but its implementation could be considered for patients with CAD, and high adrenergic activity. Absence of a significant association between baseline alphablocker therapy and critical hemodynamic incidents during anesthesia does not support the necessity of their routine preoperative administration. However, these assumptions should be confirmed through prospective controlled trials.

The study has several limitations. First, the retrospective design of the study limits the validity of the obtained results. Second, 50 patients are a relatively small cohort. However, it can be considered a representative of the population contemplating that pheochro- mocytoma is an extremely rare disease. Third, the baseline assessment of catecholamine metabolites was not standardized, which could have influenced the results of the regression analysis. Lastly, troponin measurements for assessing perioperative myocardial injury were not performed, which limited the ability to explore the relationship between baseline CAD and perioperative requirement of vasopressor support.

In conclusion, baseline SVRI is associated with perioperative requirement of vasopressor drugs, but history of CAD is a stronger prognostic factor for vasopressor support. Perioperative requirement in vasodilators is associated with baseline adrenergic activity. The evaluation of indications for extensive hemodynamic monitoring and its role in enhancing patient safety should be validated through further prospective trials.

\section{Conflict of Interest}

The authors declare that they have no conflict of interest.

\section{References}

[1] Bajwa S, Bajwa S. Implications and considerations during pheochromocytoma resection: A challenge to the anesthesiologist. Indian J Endocrinol Metab 2011; 15: 337

[2] Livingstone M, Duttchen $K$, Thompson J et al. Hemodynamic stability during pheochromocytoma resection: Lessons learned over the last two decades. Ann Surg Oncol 2015; 22: 4175-4180

[3] Naranjo J, Dodd S, Martin YN. Perioperative management of pheochromocytoma. J Cardiothorac Vasc Anesth 2017; 31: 1427-1439

[4] Groeben H, Walz MK, Nottebaum B] et al. International multicentre review of perioperative management and outcome for catecholamine-producing tumours. Br J Surg 2020; 107: e170-e178

[5] Grocott HP. Safety assessments in the avoidance of preoperative $\alpha$-receptor blockade in phaeochromocytoma surgery: the pitfalls of a zero numerator. Br J Anaesth 2017; 119: 545-546

[6] Schimmack S, Kaiser J, Probst P et al. Meta-analysis of $\alpha$-blockade versus no blockade before adrenalectomy for phaeochromocytoma. $\mathrm{Br}$ J Surg 2020; 107: e102-e108

[7] Weingarten TN, Welch TL, Moore TL et al. Preoperative levels of catecholamines and metanephrines and intraoperative hemodynamics of patients undergoing pheochromocytoma and paraganglioma resection. Urology 2017; 100: 131-138

[8] Gaujoux S, Bonnet S, Lentschener C et al. Preoperative risk factors of hemodynamic instability during laparoscopic adrenalectomy for pheochromocytoma. Surg Endosc 2016; 30: 2984-2993

[9] Wolf KI, Santos JRU, Pacak K. Why take the risk? We only live once: the dangers associated with neglecting a pre-operative alpha adrenoceptor blockade in pheochromocytoma patients. Endocr Pract 2019; 25: 106-108

[10] Gaies MG, Gurney JG, Yen AH et al. Vasoactive-inotropic score as a predictor of morbidity and mortality in infants after cardiopulmonary bypass. 2010; $11: 234-238$

[11] Koponen T, Karttunen J, Musialowicz T et al. Vasoactive-inotropic score and the prediction of morbidity and mortality after cardiac surgery. $\mathrm{Br}$ J Anaesth 2019; 122: 428-436

[12] Walz MK, Peitgen K, Walz MV et al. Posterior retroperitoneoscopic adrenalectomy: Lessons learned within five years. World J Surg 2001; 728-734 
[13] Grocott HP. Perioperative considerations in the management of pheochromocytoma: The details behind the changing dogma. J Cardiothorac Vasc Anesth 2018; 32: e11-e12

[14] Rao N, Ramachandran R, Tandon N et al. Surgical and hemodynamic outcomes in pheochromocytoma surgery: a prospective cohort study. Urology 2016; 98: 103-106

[15] Hemodynamic monitoring lessons from the ICU. Michael R. Pinsky, Jean-Louis Teboul J-L, Vincent J-L. Eds Berlin: Springer; 2019

[16] Perioperative hemodynamic monitoring and goal directed therapy: From theory to practice Cannesson M, Pearse R. Eds Cambridge: Cambridge University Press; 2014
[17] Groeben H, Nottebaum B], Alesina PF et al. Perioperative $\alpha$-receptor blockade in phaeochromocytoma surgery: An observational case series. Br J Anaesth 2017; 118: 182-189

[18] Tufton N, Gunganah K, Hussain S et al. Alpha blockade - not to be underdone. Clin Endocrinol (Oxf) 2017; 86: 306-308

[19] Challis BG, Casey RT, Simpson HL et al. Is there an optimal preoperative management strategy for phaeochromocytoma/paraganglioma? Clin Endocrinol (Oxf) 2017; 86: 163-167 